\title{
Paroxetine induced bruxism, treated with Buspirone : An unexplored entity
}

\author{
Seshadri Sekhar Chatterjee ${ }^{1}$, Arnab Maji ${ }^{2}$, Nitu Mallik ${ }^{1}$ \\ ${ }^{1}$ Department of Psychiatry, Medical College, Kolkata \\ ${ }^{2}$ Department of Pulmonology, NRSMCH, Kolkata
}

ABSTRACT :

SSRI are the widely prescribed first line drugs for depression and as depression very commonly presents with insomnia SSRI are good at improving the sleep problems also. But though rare the occurrence of sleep related side effects like bruxism,it may cause significant distress and cause of nonadherence which easily may be overlooked. In this case we report a case of Paroxetine induced bruxism and subsequently treated with withdrawing the drug and buspirone. Though the aetiology of such bruxism remained unclear, the imbalances in dopaminergic and serotonergic activities in the central nervous system is mostly implicated.

Key Words : Bruxism, Escitalopram, Parafunctional activity, Selective Serotonin Reuptake Inhibitors

\section{CASE REPORT}

A 32 year old man was presented with complaints of low mood, lethargy,weekness, weakness, anhedonia and feelings of guilt, decreased sleep with last 6 months.

He was born out of nonconsagunous marriage, educated up to class eleven, married with no significant substance abuse. Mental status examination (MSE) revealed stooped posture, poor eye contact; depressed, constricted affect; slow, soft, monotonous speech.

He was diagnosed a case of 'Severe depressive episode without psychotic syndrome' [F32.2] according to ICD $10^{[1]}$ by the practicing physician. On HAM-D (Hamilton Depression) ${ }^{[2]}$ Rating Scale the score was 22 which means severe depression.

Correspondence address :

Seshadri Sekhar Chatterjee

Mail :drssschatterjee@gmail.com
The laboratory investigations of routine hematology, blood sugar, liver function test and thyroid function test were found to be normal. A regimen of paroxetine was started with a bedtime dose of $10 \mathrm{mg}$. After 7 days at this dosage with no reported adverse effects, the dosage was increased to $20 \mathrm{mg}$ / day. After 2 weeks it changed to Paroxetine extend release $37.5 \mathrm{mg}$.

At follow-up after 1month his low mood improved with decreased anxiety and nervousness, sleep pattern was normalized.

After 45 days the patient reported excessive grinding of teeth followed by clenching of the jaw which was not present during the initial weeks of the therapy. He complained of having aching jaw muscles. A grinding or taping noise during sleep was detected by his wife, which was reportedly so loud and unpleasant that kept her awake. Lip biting and cheek biting were also reported. The dental examination did not reveal any abnormalities. 
Hence we stopped paroxetin and his problem slightly decreased. Patient was put on buspirone $5 \mathrm{mg}$ per night which eventually increased to 10 $\mathrm{mg}$ at night. Bruxism was found to be resolved within 2 weeks of dechallenge of the regimen. No rechallenge was attempted by the physician. A temporal relationship between Escitalopram and Bruxism was thus established.

Naranjo scale ${ }^{[3]}$ gave a score of 7 which denotes probable Adverse Drug Reaction.

The patient was on desvenlafaxine $50 \mathrm{mg}$ and buspirone $10 \mathrm{mg}$ and no further symptoms noted till during 3 months followup.

\section{DISCUSSION \& CONCLUSION}

Bruxism is characterized by excessive grinding of the teeth and/or excessive clenching of the jaw. Bruxism is a common problem with its prevalence ranging from $8-31 \%$ in the general population. ${ }^{[4]}$ Several symptoms are commonly associated with bruxism, including hypersensitive teeth, aching jaw muscles, orofacial pain and headaches. If severe bruxism may lead to serious periodontal damage, temporomandibular dysfunction, sleep disturbances, jaw pain, and stiffness. As a result, such cases must be treated with medication. ${ }^{[5]}$

It may be of two types- nocturnal bruxism (sleep bruxism) and diurnal bruxism (awake bruxism). The symptoms of sleep bruxism tend to be worst on waking and improve during the course of the day while the symptoms of awake bruxism may not be present at all on waking, and then worsen over the day. The exact mechanism of SSRI-induced bruxism remains unclear. Many theories have been proposed, including sleep disturbance, serotonergic-mediated inhibition of dopamine manifesting as akathisia, and SSRI-induced anxiety. ${ }^{[6]}$

Recent findings and literature have suggested that certain psychotropics, such as drugs in the selective serotonin reuptake inhibitor (SSRI), antipsychotic, and general antidepressant classes, have been implicated in inducing this condition as a secondary side effect in patients taking these medications. ${ }^{[7,8]}$

The neurochemical mechanism underlying the development of bruxism remains unclear. However, recent literatures and investigations have suggested that the central dopaminergic system (especially within the mesocortical tract), which controls muscular or motor activity, may be involved in the pathophysiology of bruxism.[9] It has been hypothesized that the mechanism for SSRI-induced bruxism may involve excessive serotonergic action on the mesocortical neurons arising from the ventral tegmental area. This action leads to a dopaminergic deficit, which causes a specific form of akathisia and akathisia-like movement of the jaw muscles, thereby leading to bruxism. ${ }^{[10]}$

Various documented case studies have reported that patients experienced drug-induced bruxism after being prescribed an SSRI to treat depressive disorders. In one case study, a 20-year-old woman prescribed paroxetine to treat her diagnosed condition of depression experienced gritting of the teeth and intense jaw tenseness, conditions that are cardinal signs of bruxism and temporomandibular joint dysfunction. ${ }^{[1]}$ A comprehensive search of tertiary resources in the Annals of Pharmacotherapy linked SSRIs to 127 published reports of SSRI-induced movement disorders, which were precursors to bruxism. ${ }^{[12]}$ The antipsychotic haloperidol has also been linked to inducing bruxism. A 40-year-old male with a history of chronic paranoid schizophrenia and a 31-year-old female diagnosed with acute psychosis reported incidences of jaw clenching and contractions consistent with bruxism. [13] Venlafaxine and fluoxetine ${ }^{[14]}$ has also been linked to this condition. These drugs, indicated to treat mood disorders, caused this unwanted side effect, which is usually treated by discontinuing the suspected psychotropic culprit.

Buspirone is an agonist of the 5-HT1A receptor that increases dopaminergic neuron firing in the ventral tegmental area and increases the synaptic release of 


\section{Paroxetine induced bruxism}

dopamine in the prefrontal cortex. ${ }^{[10]}$ These effects ameliorate drug-induced bruxism.

\section{REFERENCES}

1. World Health Organization. The ICD-10 classification of mental and behavioural disorders-diagnostic criteria for research. Geneva : World Health Organization, 1992.

2. Hamilton M. Development of a rating scale; for primary depressive illness. Br J Soc Clin Psychol. 1967 Dec; 6(4) : 278-96.

3. Naranjo CA, Busto U, Sellers EM et al. (1981). "A method for estimating the probability of adverse drug reactions". Clin. Pharmacol. Ther. 30 (2) : 239-45.

4. Manfredini D, Winocur E, Guarda-Nardini L, Lobbezoo F. Epidemiology of bruxism in adults: a systematic review of the literature. Journal of orofacial pain, 2013; 27 (2) : 99-110.

5. Attansio R. An overview of bruxism and its management. Dent Clin North Am. 1997; 41 : 229-41.

6. Romanelli F,Adler DA,Bungay KM. Possible paroxetineinduce bruxism. ANN Pharmacology. 1996 Nov; 30 (11); $1246-8$

7. Wise M. Citalopram-induced bruxism. Br J Psychiatry 2001; $178: 182$.
8. Ellison JM, Stanziani P. SSRI-associated nocturnal bruxism in four patients. J Clin Psychiatry, 1993; 54 : 432-434.

9. Lavigne GJ, Kato T, Kolta A, Sessle BJ. Neurobiological mechanisms involved in sleep bruxism. Crit Rev Oral Biol Med, 2003; $14: 30-46$.

10. Jaffee MS, Bostwick JM. Buspirone as an antidote to venlefaxine-induced bruxism. Psychosomatics, 2000; $41: 535-36$

11. Romanelli F, Adler DA, Bungay KM. Possible paroxetineinduced bruxism. Ann Pharmacother, 1996; 30 : 1246-1248.

12. Gerber PE, Lynd LD. Selective serotonin-reuptake inhibitorinduced movement disorders. Ann Pharmacother, 1998; 32:692-698.

13. Amir I, Hermesh H, Gavish A. Bruxism secondary to antipsychotic drug exposure : a positive response to propranolol. Clin Neuropharmacol, 1997; 20 : 86-89.

14. Sabuncuoglu O, Ekinci O, Berkem M. Fluoxetine-induced sleep bruxism in an adolescent treated with buspirone : a case report. Sec Care Dentist. 2009 Sept-Oct; 29(5) : 215-7

Funding sources : None

Conflict of interest statement : The author declares that there are no conflicts of interest. Informed Consent : Taken 